\title{
Developments for collective Thomson scattering equipment with a sub-THz gyrotron in LHD
}

\author{
Teruo Saito ${ }^{1, *}$, Yoshinori Tatematsu ${ }^{1}$, Yuusuke Yamaguchi ${ }^{1}$, Masafumi Fukunari ${ }^{1}$, Takumi Hirobe ${ }^{1}$, Ryushi Shinbayashi ${ }^{1}$, \\ Shunsuke Tanaka ${ }^{1}$, Kunizo Ohkubo ${ }^{1}$, Shin $\mathrm{Kubo}^{2}$, Takashi Shimozuma ${ }^{2}$, Kenji Tanaka ${ }^{2}$ and Masaki Nishiura ${ }^{3}$ \\ ${ }^{1}$ Research Center for Development of Far-Infrared Region, University of Fukui, 3-9-1 Bunkyo, Fukui city, 910-8507, Japan \\ ${ }^{2}$ National Institute for Fusion Science, National Institute of Natural Sciences, 322-6 Oroshi cho, Toki city, 509-5292, Japan \\ ${ }^{3}$ Graduate School of Frontier Sciences, The University of Tokyo, 5-1-5 Kashiwanoha, Kashiwa city, 277-8561, Japan
}

\begin{abstract}
Plan of collective Thomson scattering (CTS) experiment for the Large Helical Device (LHD) in NIFS with a $303 \mathrm{GHz}$ gyrotron is under way. Use of a sub-THz gyrotron expands the CTS-applicable region of plasma parameters. In LHD, sub-THz CTS can be applied to the high density operation region, plasmas with impurity hole, etc. Moreover, sub-THz CTS is expected to be free from ECE noise. Its "collective" use with $77 \mathrm{GHz}$ and $154 \mathrm{GHz}$ CTS will compose a powerful diagnostic system. A high power sub-THz gyrotron with a frequency of $303 \mathrm{GHz}$ has been developed. Its maximum power is $320 \mathrm{~kW}$. It oscillates in pulse mode and the maximum pulse width is around $100 \mu \mathrm{s}$, which is sufficient for use in CTS experiments. A whispering gallery mode $\mathrm{TE}_{22,2}$ was adopted for this gyrotron to avoid mode competition. Careful frequency measurement has proved purely single mode oscillation of the TE22,2 mode including turn-on and turn-off phases of the oscillation pulse. This is consistent with mode competition calculations taking account of a finite voltage rise time. A low loss transmission line is necessary for CTS. We have two possibilities. One is a new line with 1.25 inch corrugated waveguides that are optimized for the $300 \mathrm{GHz}$ band. Transmission test with the $303 \mathrm{GHz}$ gyrotron has been carried out and a sufficiently low loss coefficient has been confirmed. The other is to use an existing line with 3.5 inch corrugated waveguides for lower frequencies such as $77 \mathrm{GHz}$ and $154 \mathrm{GHz}$. Transmission test has been carried out with the $303 \mathrm{GHz}$ gyrotron and a sufficiently low loss coefficient has been confirmed also for 3.5 inch corrugated waveguides. An existing line with 3.5 inch corrugated waveguides will be used in the initial phase of $303 \mathrm{GHz}$ CTS experiment.
\end{abstract}

\section{Introduction}

CTS diagnostics using gyrotrons for electron heating around $100 \mathrm{GHz}$ have been developed [1, 2]. Sub-THz waves are promising for CTS because they do not suffer from refraction and absorption in plasmas and noise due to strong electron cyclotron emissions (ECEs). The power source for CTS should be more than $100 \mathrm{~kW}$ to realize a suitable signal-to-noise $(\mathrm{SN})$ ratio [3]. Then, a high power sub-THz gyrotron has been developed for use in CTS diagnostics in LHD.

A second harmonic gyrotron was initially developed. It realized single mode oscillation approaching $100 \mathrm{~kW}$ at $389 \mathrm{GHz}$. However, a competing fundamental harmonic mode prevented further increase in power [4]. We subsequently developed a fundamental harmonic prototype gyrotron with a whispering gallery mode (WGM) and used it to verify the design concept for stable single-mode oscillation [5]. The same design concept to use WGM mode was adopted in the design of a practical gyrotron and fabricated for use in CTS diagnostics in the LHD.

The designed operating frequency of the practical gyrotron is $303.3 \mathrm{GHz}$, which corresponds to the minimum for the ECE for standard LHD operation [6]. The cutoff density corresponding to $303 \mathrm{GHz}$ is about $10^{21} \mathrm{~m}^{-3}$. This gyrotron can be used in the high density operation mode of LHD.

The oscillation mode is the $\mathrm{TE}_{22,2}$ mode, which is a moderately high order WGM similar to the $\mathrm{TE}_{14,2}$ mode used in the prototype gyrotron. WGM mode is suitable to satisfy requirements for mode competition avoidance and low cavity surface ohmic loss simultaneously [5]. WGM modes were used in the initial stage of the development of gyrotrons for fusion plasma heating and stable oscillations without mode competition were shown [7, 8]. Volume modes were then used to reduce the ohmic loss for the continuous wave (cw) $1 \mathrm{MW}$ class gyrotrons required for the International Thermonuclear Experimental Reactor (ITER) [9]. Because cw oscillation is not required in CTS doagnostics, the 303 $\mathrm{GHz}$ gyrotron is operated in pulse mode. WGM modes are promising for complete suppression of spurious modes, including the turn-on and turn-off phases of each oscillation pulse.

* Corresponding author: saito 2729@ fir.u-fukui.ac.jp 
A low loss transmission line is necessary for realization of CTS diagnostics because the gyrotron is located far away from LHD. The transmission line will be tens of meters long. A corrugated waveguide (CW) system is a prospective candidate of a low loss transmission line. Two types of $\mathrm{CW}$ are now considered. One is a new line with 1.25 inch CW optimized for 300 $\mathrm{GHz}$ band. The other is a 3.5 inch $\mathrm{CW}$ line already installed in LHD for electron heating with lower frequency gyrotrons.

We have first evaluated the transmission efficiency of the 1.25 inch $\mathrm{CW}$ with the $303 \mathrm{GHz}$ gyrotron power. Five $2.085 \mathrm{~m}$ long waveguides were prepared for this test $[10,11]$. An attenuation coefficient of $0.02-0.04$ $\mathrm{dB} / \mathrm{m}$ sufficiently small for use in CTS was obtained. However, theoreticaliy calculated attenuation coefficients are smaller than $0.001 \mathrm{~dB} / \mathrm{m}[12,13]$. Experimental errors in the measurement of the very small attenuation might cause the discrepancy. In addition, absorption by water vapor of the order of 0.01 $\mathrm{dB} / \mathrm{m}$ should also be considered.

Since installtion of a new line with the 1.25 inch CW is difficult for the time being, an exsiting line with 3.5 inch $\mathrm{CW}$ will be used in the initial stage of $303 \mathrm{GHz}$ CTS. Then, we have measured the transmisson efficiency with spare waveguides.

\section{Design, fabrication and test of 303 GHz gyrotron}

It was necessary to use WGM higher than that of the prototype gyrotron to extend the pulse width and the duty ratio up to $10 \%$. Preliminary design consideration was carried out to find candidate modes of the practical gyrotron. Figure 1 plots the stationary oscillation power obtained with a gyrotron design code [14] including the competition with neighboring modes. This figure indicates that WGM modes are not always appropriate for a high power oscillation. The $\mathrm{TE}_{19,2}$ and $\mathrm{TE}_{20,2}$ modes subject to competition with neighboring modes. The $\mathrm{TE}_{22,2}$ mode was finally adopted for the practical gyrotron [15]. The same mode was also employed in [16

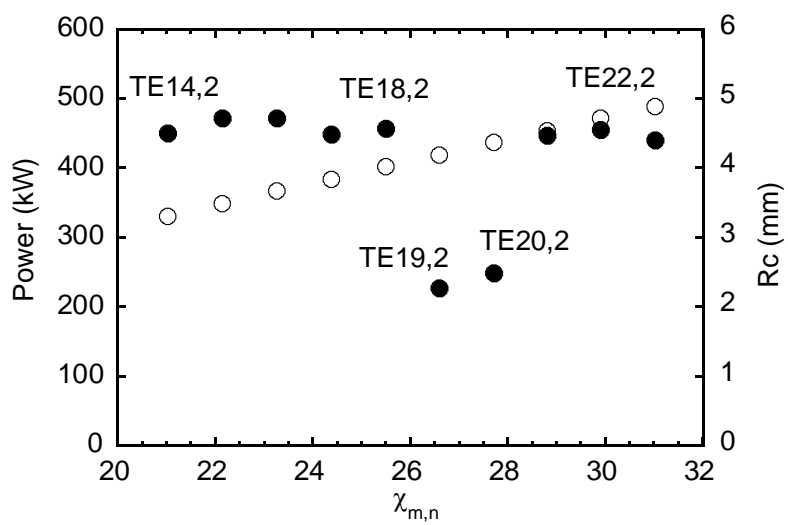

Fig. 1. Power calculation of candidate modes (solid circles). The beam voltage and beam current were set at $65 \mathrm{kV}$ and 20 A, respectively. Possible competing modes are included in the calculation. The cavity radius corresponding to the oscillation frequency of $303.3 \mathrm{GHz}$ is also shown by open circles.
- 18]. Oscillation characteristics of the $\mathrm{TE}_{22,2}$ mode were examined in detail with the gyrotron design code and stable single mode oscillation was expected. A power of more than $300 \mathrm{~kW}$ was predicted for a beam voltage $V_{k}$ of $65 \mathrm{kV}$, a beam current $I_{B}$ of $15 \mathrm{~A}$, and a velocity pitch factor $\alpha$ of 1.2. Ohmic loss on the cavity surface is safely smaller than $2 \mathrm{~kW} / \mathrm{cm}^{2}$ up to the duty ration of $10 \%$.

An electron gun was newly designed and optimized for the $\mathrm{TE}_{22,2}$ mode by using the same design principle as reported in Ref. [19]. The design predicted electron beams of high quality with the expected $\alpha$ values and sufficiently small velocity spreads up to $I_{B}=20 \mathrm{~A}$ [20].

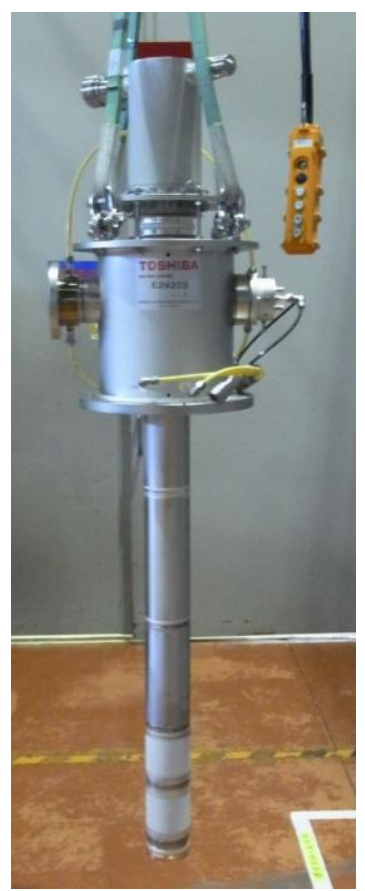

Fig. 2. Picture of the fabricated practical gyrotron. A Gaussian beam is radiated through the vacuum window at the left side.

Figure 2 is a picture of the fabricated gyrotron. This gyrotron is mounted on a liquid He-free 12 T SC magnet. The diameter of the room temperature bore of SC is 100 $\mathrm{mm}$. An internal mode converter composed of a helicalcut Vlasov type launcher and four mirrors is installed into a rather narrow room. The vacuum window is made of a c-axis cut crystal sapphire disk. Its thickness is optimized for $303.3 \mathrm{GHz}$.

Oscillation tests have been carried out and a maximum power higher than $320 \mathrm{~kW}$ for $V_{k}=65 \mathrm{kV}$ and $I_{B}=15 \mathrm{~A}$ was obtained [20, 21]. The oscillation efficiency was higher than $30 \%$. Figure 3 shows the experimentally obtained power and efficiency as functions of the beam current. The calculated values are also plotted. In the calculation, $\alpha$ values were evaluated for each experimental parameters with the E-GUN code. The experimental results are essentially consistent with calculations with $\alpha$ ranging from 1.1 to 1.3 , although the experimentally obtained powers are slightly lower than the calculated values. In this calculation instant voltage rise is assumed and interaction between the operating 


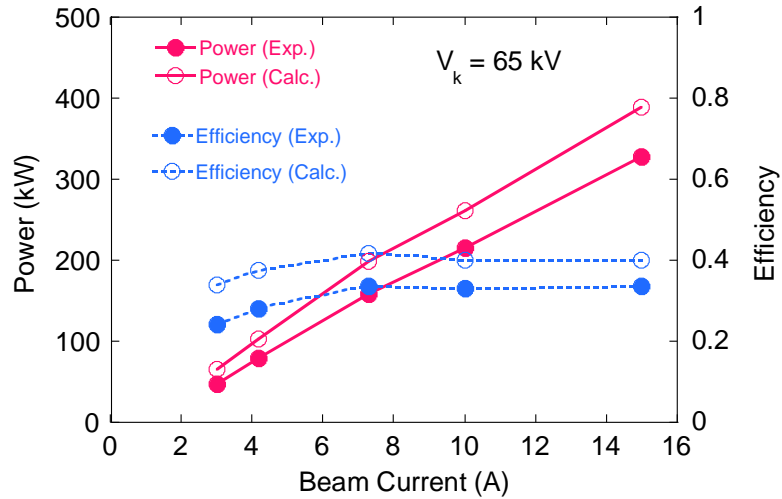

Fig. 3. Experimental and calculated values of the power and the efficiency are plotted. The TE21,2 mode is included in the calculation of the power and efficiency.

$\mathrm{TE}_{22,2}$ mode and the $\mathrm{TE}_{21,2}$ mode is included. Although many other modes exist in between the two modes, interaction between the two modes is particularly strong.

The radiation pattern was measured using an infrared camera as the increasing temperature profile on a polyvinyl chloride (PVC) plate located in front of the vacuum window. A Gaussian like radiation pattern similar to that expected from the mode converter design calculation was obtained. The radiation pattern was somewhat elliptic and a small fraction of higher order modes was observed [20].

Measurement of the oscillation frequency using a Fabry-Perot interferometer verified single-mode oscillation of the $\mathrm{TE}_{22,2}$ mode. The oscillation frequency was measured more accurately using a heterodyne receiver system. The measured frequency was 303.3 $\mathrm{GHz}$ when $V_{k}=62 \mathrm{kV}$ and the magnetic field at the cavity $B=11.59 \mathrm{~T}$. This frequency is equal to the designed $\mathrm{TE}_{22,2}$ mode frequency to within an accuracy of the order of $10 \mathrm{MHz}$.

Frequency control is very important for application to CTS because a narrow band notch filter is used. A notch filter is necessary to protect the highly sensitive receiver system from the gyrotron-frequency wave stemming from scattering off the vacuum vessel. The oscillation

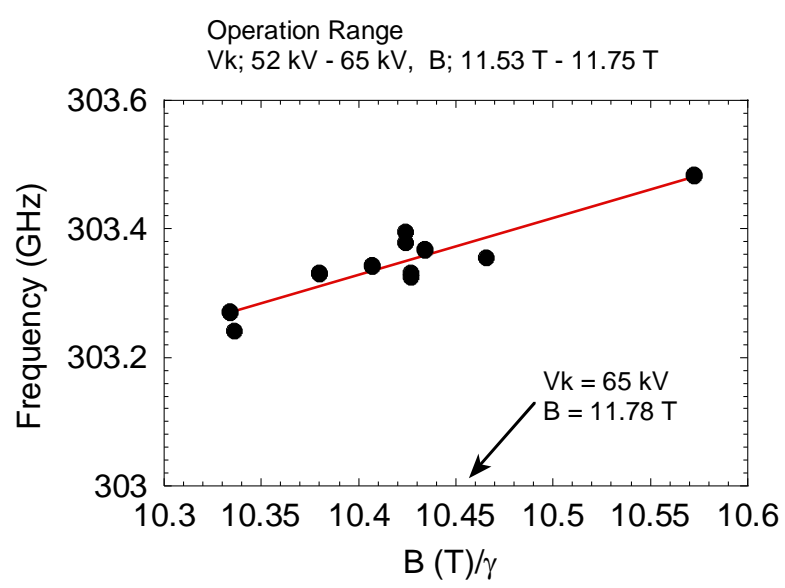

Fig. 4. Frequency variation as a function of a ratio of the magnetic fireld strength at the cavity $B$ and the relativistic factor of the electrn beam $\gamma$. frequency slightly varies with the operation parameters such as $V_{k}$ and $B$. We can control the oscillation frequency at around $303.3 \mathrm{GHz}$ by appropriately changing $V_{k}$ and $B$. Figure 4 indicates controllability of the frequency. The frequency variation with $B$ and $V_{k}$ was less than $\pm 100 \mathrm{MHz}$. The center frequency of the notch filter is $303.3 \mathrm{GHz}$ and the full notch width is 0.5 GHz. The observed frequency variation around 303.3 $\mathrm{GHz}$ is safely smaller than the rejection width. The expected frequency width of the scattered signal is much wider than the notch width [3].

The pulse width was extended up to $100 \mu \mathrm{s}$. Relevant waveforms are plotted in Fig. 5. The output power is approximately $200 \mathrm{~kW}$ for this case. The oscillation signal was measured using a pyroelectric detector in the time integration mode. The linear increase in this signal

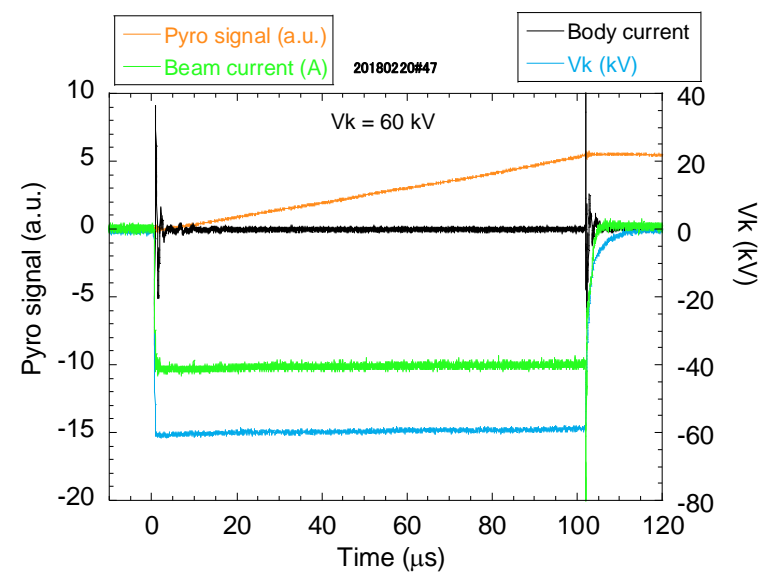

Fig. 5. Waveforms of an $100 \mu$ s oscillation. The beam voltage and beam current were set at $60 \mathrm{kV}$ and $10 \mathrm{~A}$, respectively.

indicates very stable power through the pulse width. This pulse width is sufficient for use in CTS diagnostics.

The frequency spectrum measured with the heterodyne receiver system was very narrow and stable across the pulse width. The frequency variation in one pulse is smaller than $10 \mathrm{MHz}$ and the pulse to pulse difference is less than $20 \mathrm{MHz}$ [15]. Parasitic modes were searched for over a wide frequency band, ranging from $290 \mathrm{GHz}$ to $312 \mathrm{GHz}$, using the same heterodyne receiver system. No oscillation other than the $\mathrm{TE}_{22,2}$ mode was observed throughout the pulse width, including during the turn-on and the turn-off phases. The dynamic range of measurement of about $40 \mathrm{~dB}$ is larger than the power range of the expected scattered signal [3].

The voltage of the modulation anode of the triode electron gun was set with a simple resistive divider. Stable and no-parasitic-mode oscillation without sophisticated voltage control of the modulation anode is likely due to a very fast rise time of the beam voltage. The beam voltage attains to $65 \mathrm{kV}$ in about $0.25 \mu \mathrm{s}$. A fast semiconductor switch realizes this short rise time. Suppression of parasitic modes for a voltage rise time shorter than $1 \mu$ s was predicted [22]. A theoretical study just corresponding to the present gyrotron also predicts single mode oscillation of the $\mathrm{TE}_{22,2}$ mode [23]. The frequency mismatch of the $\mathrm{TE}_{22,2}$ mode is zero or even negative when the beam voltage starts to increase. 
Analysis with a non-stationary axial field structure shows that a backward wave of the same transverse mode as the design mode is first soft-self excited and gradually transits to the high power gyrotron mode. This was predicted in [24].

\section{Transmission test of 3.5 inch corrugated waveguides [25]}

For CTS in LHD, it is necessary to transmit the gyrotron power to LHD with a low loss transmission line composed of corrugated waveguides over about $60 \mathrm{~m}$. At the first stage of the $300 \mathrm{GHz}$ CTS experiment, an existing 3.5 inch $\mathrm{CW}$ line is used for power transmission. Since this waveguide is not optimized for $300 \mathrm{GHz}$ but for lower frequency gyrotrons for electron heating. Thus, transmission test is necessary. The corrugation parameters of the 3.5 inch CW are shown in Table I. The Bragg frequency of this waveguide is $187 \mathrm{GHz}$.

Table 1. Corrugation parameters of 3.5 in waveguide.

\begin{tabular}{|c|c|}
\hline Waveguide diameter $: \mathrm{a}$ & $88.9 \mathrm{~mm}$ \\
\hline Corrugation depth: $\mathrm{d}$ & $0.60 \mathrm{~mm}$ \\
\hline Corrugation width: $\mathrm{w}$ & $0.60 \mathrm{~mm}$ \\
\hline Corrugation Pitch: $\mathrm{p}$ & $0.80 \mathrm{~mm}$ \\
\hline
\end{tabular}

The output beam from the gyrotron is not axisymmetric and expands along propagation. Using a Matching Optics Unit (MOU), the output beam from the gyrotron was shaped into a Gaussian beam that couples to the $\mathrm{HE}_{11}$ mode of the waveguide with a high coupling coefficient [26]. Figure 6 indicates setup of MOU. It is composed of two elliptic mirrors to tailor the beam radiated from the gyrotron to a Gaussian beam with the waist at the waveguide entrance position and two plane mirrors to adjust the beam direction.

The beam shape near the design waist position was

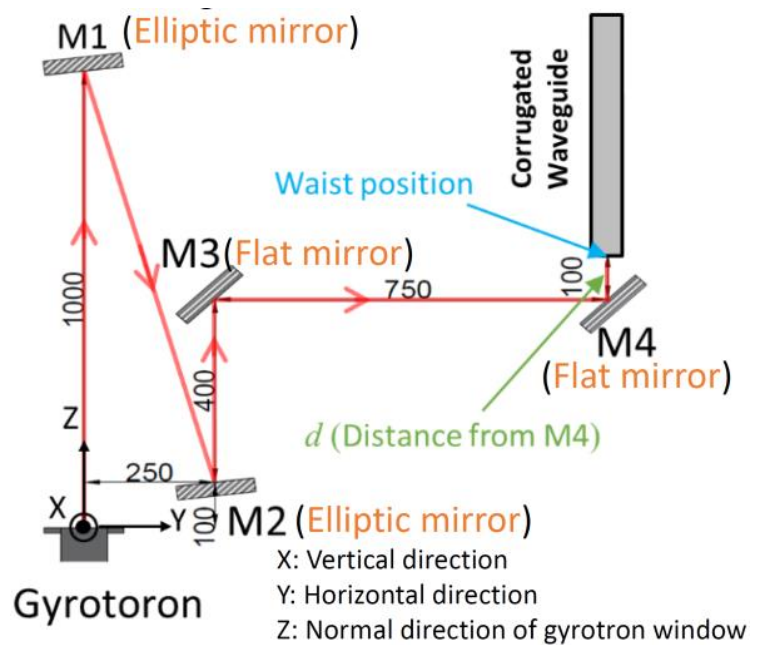

Fig. 6. Setup of Matching Optics Unit (MOU).

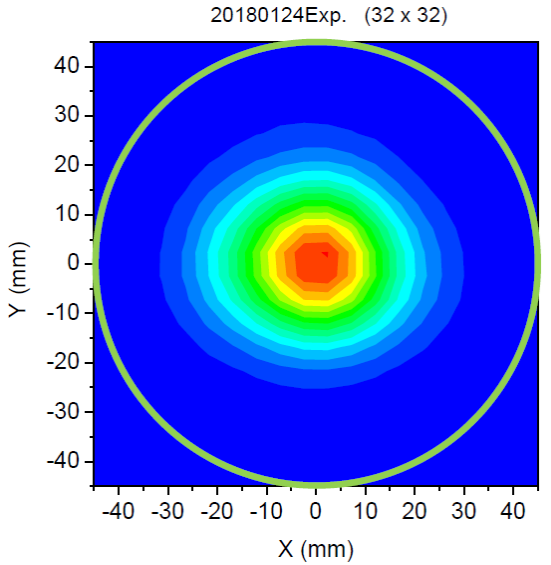

Fig. 7. Beam shape at the entrance position of 3.5 corrugated waveguide.

slightly elliptical. This is possibly because the gyrotron beam contains higher order modes. Figure 7 represents the beam shape at the waveguide entrance position measured as the distribution of temperature increase on a PVC plate. The green circle represents the waveguide cross section.

Figure 8 is a picture of the transmission test. Ten one-meter long waveguides were connected straight on a tight frame. The relative transmitted power was

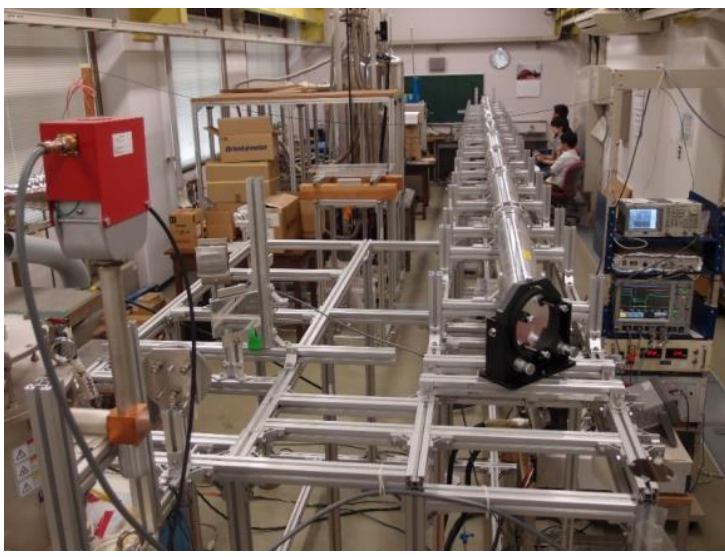

Fig. 8. Picture of transmission test.

evaluated at every $2 \mathrm{~m}$ distance by integrating the distribution of the temperature increase on a PVC plate over the waveguide cross section. The PVC plate was located just at the out pout position for each distance measurement.

Figure 9 plots the relative power as a function of the transmission distance along 3.5 inch $\mathrm{CW}$. Although measurements includes some error, attenuation is very low probably smaller than $0.01 \mathrm{~dB} / \mathrm{m}$. Correspondingly, the transmission loss is smaller than $0.6 \mathrm{~dB}$ for $60 \mathrm{~m}$.

Figure 9 represents the total power transmitted to each measurement position. It includes attenuation of higher order modes excited in waveguides. In fact, owing to excitation of higher order modes, very complicated structures were observed at each measurement point.

Then, nonlinear mode content analysis was carried out with the same method as shown in [27]. In this 


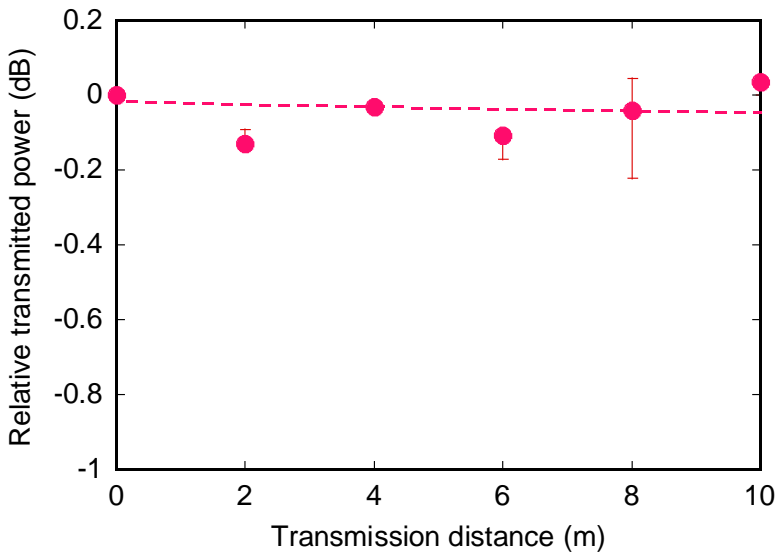

Fig. 9. Total power transmitted to each measurement position.
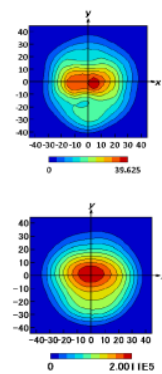

20011105
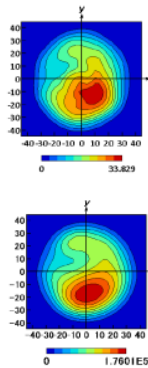
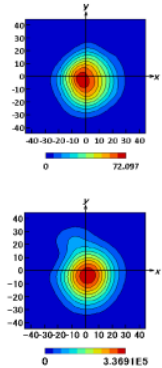
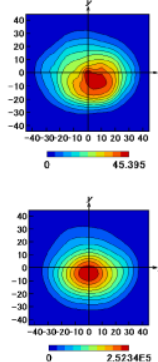
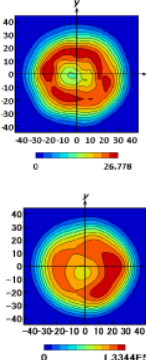

Fig. 10. Comparison between observed pattern of power distribution at each measured position (upper row) and the composed power distribution with calculated power rfactions and phases (lower row).

analysis, 19 higher order modes were taken into account. Figure 10 represents the result of the analysis. Calculated power distributions as the composition of the $\mathrm{HE}_{11}$ mode and higher order modes well reproduces the measured power distribution. This composition takes account of relative phases between modes. The power fraction of $\mathrm{HE}_{11}$ mode was about $82 \%$. Main higher order modes were co polarizing $\mathrm{HE}_{21}$ mode and $\mathrm{HE}_{12}$ mode with power fractions around $4 \%$.

The transmission test shows that the existing 3.5 inch waveguide line can be used for $300 \mathrm{GHz}$ CTS experiment. In the CTS experiment, more axisymmetric beam will be coupled into the waveguide.

\section{Summary}

Sub THz CTS experiment is under preparation with a pulse gyrotron in the $300 \mathrm{GHz}$ band with collaboration between FIR UF and NIFS.

The design concept of the practical gyrotron is the same as that used in the prototype gyrotron. Higher mode TE22,2 was adopted to reduce Ohmic loss on the cavity surface. Stable single mode oscillation has been confirmed and the oscillation power and frequency are very stable over the pulse width. No parasitic mode was observed during the turn-on and turn-off phases. This is probably due to a finite voltage rise time. This gyrotron can be used in CTS diagnostics in LHD.

For power Transmission, an existing transmission line with 3.5 inch corrugated waveguides is used at the first stage. Although this waveguide is not optimized for $300 \mathrm{GHz}$ band, transmission test with the gyrotron have shown very low transmission loss.

Sub THz CTS experiment will start soon. Its "collective" use with $77 \mathrm{GHz}$ and $154 \mathrm{GHz}$ CTS will compose a powerful diagnostic system in LHD.

\section{References}

1. Bindslev et al., Phys. Rev. Lett. 97, 205005 (2006).

2. M. Nishiura et al., Nucl. Fusion 54, 023006 (2014).

3. T. Saito et al., Journal of Phys.: Conference Series 227, 012013 (2010).

4. T. Saito et al., Phys. Plasmas 19, 063106 (2012).

5. Y. Yamaguchi et al., Nucl. Fusion, 55, 013002 (2015).

6. T. Notake et al., Rev. Sci. Instrum. 79, 10E732 (2008).

7. K. E. Kreischer et al., Phys. Fluids B2, 640 (1990).

8. A. Kasugai et al., Fusion Eng. Design 26, 281 (1995).

9. K. Sakamoto et al., Nucl. Fusion 49, 095019 (2009).

10. T. Saito et al., IRMMW-THz 2016, H5P.16.03, Copenhagen, Sep. 25-30 (2016).

11. T. Hirobe et al., Plasma Conference 2017, 21P-53, Himeji, Nov. 20-24 (2017).

12. J. Doane, Infrared and Millimeter Waves, Ed. K. J. Button, Academic Press, vol.13, pp. 123-170, 1985.

13. E. A. Nanni et al., J. Infrared Milli Terahz Waves 33, 695 (2012).

14. Y. Tatematsu et al. J Infrared Milli Terahz Waves 33, 292 (2012).

15. T. Saito et al., IRMMW-THz 2017, RA2.1, Cancun, Aug 27 - Sept 1 (2017).

16. K. Felch et al., Conf. Digest of $17^{\text {th }}$ Int. Conf. Infrared and Millimeter Waves, p.184, Pasadena, Dec. 14-17 (1992).

17. K. Sakamoto et al., ibid, p.188, Pasadena, Dec. 14-17 (1992).

18. K. Sakamoto et al., Phys. Rev. Lett. 73, 3532 (1994).

19. Y. Yamaguchi et al., Phys. Plasmas 19, 113113 (2012).

20. Y. Yamaguchi et al., J. Instrumentation 10, C10002 (2015).

21. T. Saito et al., Plasma Fusion Res. 12, 1206013 (2017).

22. G. S. Nusinovich et al., Phys. Plasmas 15, 103101 (2008).

23. O. Dumbrajs et al., Phys. Plasmas 23, 023106 (2016).

24. G. S. Nusinovich et al., Phys. Rev. Lett. 96, 125101 (2006).

25. R. Shinbayashi et al., Plasma Conference 2017, 21P-55, Himeji, Nov. 20-24 (2017).

26. J. P. Crenn, Appl. Optics 23, 3428 (1984).

27. K. Ohkubo et al., J Infrared Milli Terahz Waves 38, 853 (2017). 